\title{
Dosimetric considerations and early clinical experience of accelerated partial breast irradiation using multi-lumen applicators in the setting of breast augmentation
}

\author{
Mani Akhtari, MD', Ramiro Pino, PhD², Sarah B. Scarboro, PhD², Barbara L. Bass, MD³, Darlene M. Miltenburg, MD4, \\ E. Brian Butler, MD², Bin S. Teh, MD² \\ 'Department of Radiation Oncology, University of Texas Medical Branch, Galveston, TX, 2Department of Radiation Oncology, Houston \\ Methodist Hospital, Cancer Center and Research, Institute, Houston, TX, ${ }^{3}$ Department of Surgery, Houston Methodist Hospital, Houston, TX, \\ ${ }^{4}$ Texas Women's Comprehensive Breast Center, Houston, TX, USA
}

\begin{abstract}
Purpose: Accelerated partial breast irradiation (APBI) is an accepted treatment option in breast-conserving therapy for early stage breast cancer. However, data regarding outcomes of patients treated with multi-lumen catheter systems who have existing breast implants is limited. The purpose of this study was to report treatment parameters, outcomes, and possible dosimetric correlation with cosmetic outcome for this population of patients at our institution.

Material and methods: We report the treatment and outcome of seven consecutive patients with existing breast implants and early stage breast cancer who were treated between 2009 and 2013 using APBI following lumpectomy. All patients were treated twice per day for five days to a total dose of $34 \mathrm{~Gy}$ using a high-dose-rate ${ }^{192} \mathrm{Ir}$ source. Cosmetic outcomes were evaluated using the Harvard breast cosmesis scale, and late toxicities were reported using the Radiation Therapy Oncology Group (RTOG) late radiation morbidity schema.

Results: After a mean follow-up of 32 months, all patients have remained cancer free. Six out of seven patients had an excellent or good cosmetic outcome. There were no grade 3 or 4 late toxicities. The average total breast implant volume was $279.3 \mathrm{cc}$, received an average mean dose of $12.1 \mathrm{~Gy}$, and a maximum dose of 234.1 Gy. The average percentage of breast implant volume receiving $50 \%, 75 \%, 100 \%, 150 \%$, and $200 \%$ of the prescribed dose was $15.6 \%, 7.03 \%$, $4.6 \%, 1.58 \%$, and $0.46 \%$, respectively. Absolute volume of breast implants receiving more than $50 \%$ of prescribed dose correlated with worse cosmetic outcomes.

Conclusions: Accelerated partial breast irradiation using a multi-lumen applicator in patients with existing breast implants can safely be performed with promising early clinical results. The presence of the implant did not compromise the ability to achieve dosimetric criteria; however, dose to the implant and the irradiated implant volume may be related with worse cosmetic outcomes.
\end{abstract}

Key words: APBI, brachytherapy, breast augmentation, Contura, SAVI ${ }^{\circledR}$.

\section{Purpose}

Multiple randomized trials over the last four decades have established the equivalence of mastectomy and breast-conserving therapy (BCT) in early stage breast cancer [1]. Breast-conserving therapy involves lumpectomy followed by adjuvant radiation therapy. More recently, accelerated partial breast irradiation (APBI) has become an acceptable means of delivering adjuvant radiation in early stage breast cancer patients who meet certain favorable criteria $[2,3]$. The benefits of APBI include shorter treatment times, sparing of a larger amount of normal tissue, and focal irradiation of the area highest at risk of recurrence around the tumor bed [4]. Initially, APBI consisted of interstitial catheter placement either via a template based approach or ultrasound guided free-hand approach [5] but these both required extensive expertise in their placement. After approval of MammoSite ${ }^{\circledR}$ (Hologic Inc, Bedford, MA, USA) in 2002 and creation of newer multi-lumen devices such as Contura ${ }^{\circledR}$ (Hologic Inc, Bedford, MA, USA) and SAVI ${ }^{\circledR}$ (Cianna Medical, Aliso Viejo,
Address for correspondence: Prof. Bin S. Teh, MD, Department of Radiation Oncology, Houston Methodist Hospital Cancer Center, 6565 Fannin, Ste \#DB1-077, Houston, Texas 77030, USA, phone: +1 713-441-4800, fax: +1 713-441-4493, 凶 e-mail: bteh@houstonmethodist.org
Received: 02.09 .2015

Accepted: 08.11 .2015

Published: 30.12 .2015 
CA, USA), the rates of utilization of brachytherapy-based APBI (B-APBI) have continued to rise and now approach $11 \%$ in patients older than 50 years old who have undergone BCT [6]. Multiple consensus guidelines have been developed to specify the appropriate candidates for B-APBI $[7,8,9,10]$. However, none of these address the role or feasibility of B-APBI in patients with existing breast implants who wish to undergo BCT. Given that now nearly 286,000 women undergo breast augmentation annually, an increase of 35\% from 2000 to 2012, the rates of patients with pre-existing implants who will be diagnosed with breast cancer will continue to rise [11] Therefore, it is imperative to understand the clinical implications of B-APBI in this patient population.

The reported experience with B-APBI in the setting of breast augmentation is limited $[12,13]$, with no known specific dose-constraints for breast implants to date. We had also previously reported the long-term outcome and dosimetric considerations in a patient with pre-existing breast implants who was treated with B-APBI [14]. The present study expands on our work by examining the outcome and dosimetric evaluation of all the patients with breast augmentation who have been treated in our institution with B-APBI. To our knowledge, this is the first such reported series with early clinical outcomes in this patient population and detailed examination of the dosimetric parameters of breast implants in B-APBI.

\section{Material and methods}

After obtaining appropriate Institutional Review Board approval, we examined the database of patients who had undergone B-APBI at our institution from 2007 to 2013 and identified seven patients with existing breast implants at the time of surgery and throughout treatment. All patients had undergone lumpectomy prior to radiation treatment. Due to the complexity of device placement adjacent to the breast implants, the breast surgeons placed the devices. Each patient was treated using either a Contura or SAVI device, and all of the devices were placed via a closed cavity approach. The planning computed tomography (CT) simulation was performed 48-72 hours following device placement in our department (Brilliance Big Bore, Philips Healthcare, Andover, MA). All patients were simulated in the supine position, with the arm on the affected side raised over their head. A small amount of contrast $(0.5 \mathrm{cc})$ was added to the saline mixture filling the balloon for Contura patients to improve visualization of the balloon on CT.

Given the variations in multi-lumen or catheter based brachytherapy [15], the integrity, orientation and size of the device, as well as distance to skin and rib were all verified prior to treatment planning before each treatment. Treatment planning was completed using BrachyVision Treatment Planning System (Varian, Palo Alto, CA, USA). The planning target volume for evaluation (PTV_EVAL) consisted of $1 \mathrm{~cm}$ of tissue surrounding the device but limited to a distance of $5 \mathrm{~mm}$ from the skin for the Contura patients, $3 \mathrm{~mm}$ for the SAVI patients, and excluding the chest wall. We also attempted to minimize the $V_{150}$ of normal breast tissue (volume receiving $150 \%$ of prescribed dose) to $<50 \mathrm{cc}$ and $\mathrm{V}_{200}<10 \mathrm{cc}$. The volume of air and seroma within the PTV_EVAL was less than $10 \%$ and this was also verified prior to each treatment.

At the time of treatment planning, since no dose constraints were known for breast implants, no special considerations were made to exclude dose or PTV_EVAL from the implants. A total dose of $34 \mathrm{~Gy}$ was prescribed to PTV_EVAL in 3.4 Gy twice daily treatments using a high-dose-rate ${ }^{192}$ Ir source. Prior to each treatment, CT-based image guidance was used to verify the device size, position, and distance to skin. The volume of the breast implants was contoured retrospectively and dosimetric analysis were conducted based on the initial treatment plan on BrachyVision. At the time of follow-up, patients were prospectively assessed for toxicity and cosmetic outcome. Harvard breast cosmesis scale was used to evaluate cosmetic outcome, which rates the outcome from excellent to poor depending on the difference compared to the untreated breast [15]. Additionally, the patients' own personal satisfaction with their cosmetic outcome was also recorded. Follow-up pictures were obtained for visual comparison to the contralateral breast and the shape of the treated breast prior to treatment.

\section{Results}

Full characteristics of the seven patients are listed in Table 1. The average age at the time of diagnosis was 61. All of the patients had undergone lumpectomy with the final pathology revealing invasive ductal carcinoma (IDC), ductal carcinoma in situ (DCIS), or both. All cases were estrogen receptor and/or progesterone receptor positive and HER-2/neu negative. Five patients had negative margins and two had focally positive margins. The clinical stages ranged from TisN0M0 to T1cN0M0 and all patients with invasive disease had undergone sentinel lymph node biopsy. The mean duration of follow-up was 32 months. Five of the seven patients received adjuvant hormonal therapy.

At the time of last follow-up, all patients remained free of recurrence. Figure 1 shows an axial image of each patient's isodose lines along with their corresponding cosmetic outcome. Five patients had a Harvard breast cosmesis score of 1 or excellent, one patient had a score of 2 or good for difference in shape or size, and one had a score of 3 or fair for obvious difference in the size and shape of the treated breast. Based on the RTOG late radiation morbidity schema, three patients had a score of 0 , three had a score of 1 for presence of telangiectasia, hyperpigmentation or erythema, and one had a score of 2 for tender and bright erythema with moderate edema.

One of the patients had to undergo replacement of bilateral breast implants due to age-related leakage in bilateral breasts. Another patient with cosmetic outcome of 3 experienced intra and extracapsular rupture of the treated implant almost 1.5 years after completion of APBI as evidenced by breast MRI. An ultrasound of the breasts about 6 months after completion of her treatment had previously shown intact implants, therefore ruling out implant rupture due to APBI device placement. She also developed a cystic fluid collection close to her implant, 
Table 1. Clinical characteristics and outcomes of the seven patients treated with brachytherapy-based accelerated partial breast irradiation (B-APBI) with existing breast implants

\begin{tabular}{lccccccccc} 
& $\begin{array}{c}\text { Age at } \\
\text { diagnosis }\end{array}$ & Histology & $\begin{array}{c}\text { Margin } \\
\text { status }\end{array}$ & $\begin{array}{c}\text { AJCC } \\
\text { stage }\end{array}$ & Device & $\begin{array}{c}\text { Duration of } \\
\text { follow-up } \\
\text { (months) }\end{array}$ & Cosmesis & $\begin{array}{c}\text { Late } \\
\text { toxicity }\end{array}$ & $\begin{array}{c}\text { Adjuvant } \\
\text { therapy }\end{array}$ \\
\hline Patient \#1 & 71 & IDC & $\begin{array}{c}\text { Focally } \\
\text { positive }\end{array}$ & T1cNOMO & Contura & 2 & 1, Excellent & Grade 0 & Tamoxifen \\
\hline Patient \#2 & 68 & IDC & Negative & T1bNOMO & Contura & 58 & 2, Good & Grade 1 & Arimidex \\
\hline Patient \#3 & 55 & IDC & Negative & T1aNOMO & SAVI Mini 6-1 & 47 & 1, Excellent & Grade 1 & Tamoxifen \\
\hline Patient \#4 & 54 & IDC+ DCIS & $\begin{array}{c}\text { Focally } \\
\text { positive }\end{array}$ & T1bNOMO & SAVI Mini 6-1 & 48 & 1, Excellent & Grade 1 & Letrozole, \\
Exemestane
\end{tabular}

IDC - invasive ductal carcinoma, DCIS - ductal carcinoma in situ, AJCC - American Joint Committee on Cancer, $7^{\text {th }}$ edition. Cosmesis: based on the Harvard/NSABP/ RTOG cosmesis grading scale; Late toxicity based on RTOG late morbidity scoring schema; TAC - docetaxel, doxorubicin, cyclophosphamide

which was aspirated and found to be benign. The remainder of the implants remained intact through the duration of the study. At last follow-up, all patients expressed satisfaction with their cosmetic outcomes and graded the overall appearance of the treated breast as excellent or good.

The average device to breast implant surface in our population was $1.2 \mathrm{~mm}$ and the average device to skin surface was $4.4 \mathrm{~mm}$ (range 2-7.6 $\mathrm{mm}$ ). The mean volume of the PTV_EVAL amongst our patients was $55.9 \mathrm{cc}$ and an air/seroma volume of $2.9 \mathrm{cc}$. The mean percentage of the PTV_EVAL volume receiving 90\%, 95\%, and 100\% of the prescribed dose $\left(\mathrm{V}_{90}, \mathrm{~V}_{95}, \mathrm{~V}_{100}\right)$ was $97.7 \%, 95.9 \%$, and $93.1 \%$, respectively. The maximum skin dose ranged from $95.6 \%$ to $119 \%$ and the maximum rib dose $14.2 \%$ to $113.4 \%$ of the prescribed dose. The average volume of the normal breast tissue receiving $150 \%$ of the prescribed dose $\left(\mathrm{V}_{150}\right)$ and $\mathrm{V}_{200}$ was $20.1 \mathrm{cc}$ and $9.4 \mathrm{cc}$, respectively. The average total breast implant volume was $279.3 \mathrm{cc}$ and received an average mean dose of $12.1 \mathrm{~Gy}$, minimum dose of $1.9 \mathrm{~Gy}$, and a maximum dose of $234.1 \mathrm{~Gy}$. The percentage of breast implant volumes receiving 50\%, 75\%, $100 \%, 150 \%$, and $200 \%$ of the prescribed dose (implant $\mathrm{V}_{50}, \mathrm{~V}_{75}, \mathrm{~V}_{100}, \mathrm{~V}_{150}, \mathrm{~V}_{200}$ ) was $15.6 \%, 7.03 \%, 4.6 \%, 1.58 \%$, and $0.46 \%$, respectively. Table 2 displays the individual dosimetric values for each patient.

Table 3 shows the relative dose to the breast implant along with absolute volume of the implant receiving the same dose represented as $\mathrm{V}_{50}{ }^{\prime}$, etc. The two patients who did not have an excellent cosmetic outcome (\#2 and \#7) also had the highest absolute volume of their implants receiving $50 \%$ of the prescribed dose as seen in Figure 2 $\left(\mathrm{V}_{50}{ }^{\prime}\right.$ to 72.9 and $111.9 \mathrm{cc}$, respectively). The higher treated volume was also apparent across the increasing dose ranges, although not as pronounced. Patient \#7, who has now experienced implant rupture, also had the highest maximum dose to the implant (535.1 Gy) and the highest mean dose (23.1 Gy). However, patient \#2's mean and maximum dose to the implant was closer to the average of the population (15.4 Gy and 72.8 Gy, respectively).

\section{Discussion}

With the advent of B-APBI and its shorter treatment duration, an increasing number of women with breast cancer have access to adjuvant radiation therapy. Per multiple national and international guidelines, B-APBI is now considered a standard of care option in women with early stage breast cancer $[2,3,7,8,9,10]$. Although there are an increasing number of reports about the safety and efficacy of B-APBI, the appropriate patient population remains a contentious issue since only one randomized study has compared whole breast irradiation to B-APBI $[16,17]$. Furthermore, data regarding treatment of patients with existing breast implants using B-APBI is limited.

Kuske et al. were the first to report on utilization of B-APBI in the setting of breast augmentation in the abstract form [12]. Even though they had a median follow-up of 36 months and observed an excellent or good cosmetic outcome in $97 \%$ of the patients, the majority were treated with interstitial implants. Kuske et al. also recently published his interstitial technique in the presence of breast implants but no dosimetric data were reported [18]. Bloom et al. were the first to report on dosimetric parameters in a case report of a patient who had undergone treatment with B-APBI with existing breast implants but their follow-up was only 6 months post-treatment [13]. Table 4 summarizes the existing literature in comparison to our reported outcomes.

We had previously reported the longest follow-up to our knowledge at nearly 5 years post-treatment and suggested several dosimetric parameters relating to breast implants that could be of significance [14]. We have now expanded on our original report by including all of our treated patients, with the longest reported follow-up in this patient population, and moreover adds several dosimetric parameters that can be useful while treating APBI with pre-existing breast implants. All of our patients have been free of disease to this date, even patients \#1 and \#4 who had focally positive margins. It is important to note that their margin status could be somewhat misleading since it was adjacent to the breast implant and a negative 
1a



$2 a$



$3 a$

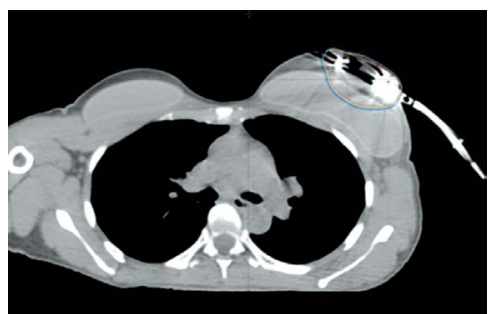

$4 a$



$5 a$



$6 a$

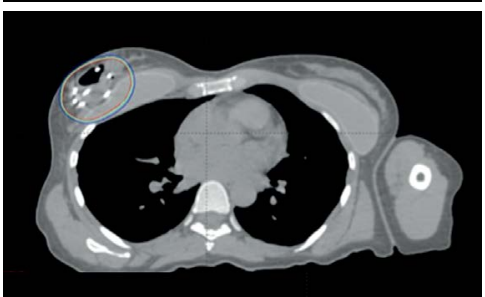

$7 a$



1b

$2 \mathrm{~b}$
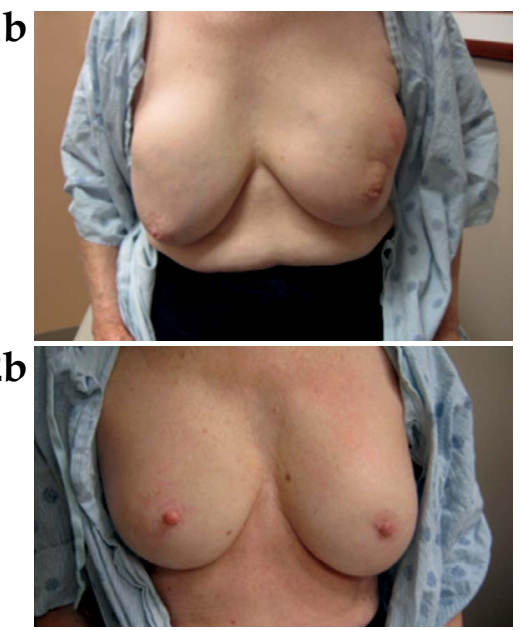

$3 b$



$4 b^{*}$

$5 b$

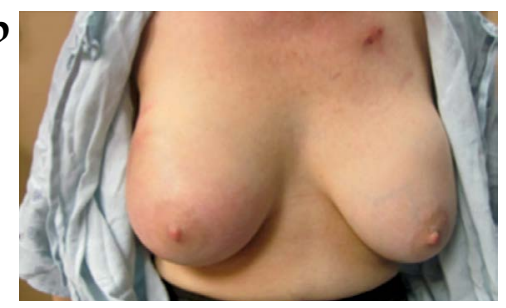

$6 b$



$7 b$

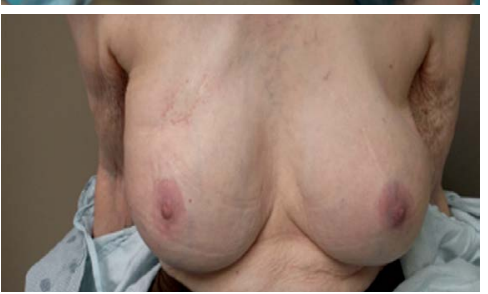

Fig. 1. Isodose lines of all seven patients along with their corresponding cosmetic outcomes. Each row corresponds to the aforementioned patient. Left column shows a representative cross-section of their respective isodose lines along with the device location. Red line represents 340 cGy (100\%), white line 323 cGy (95\%), green line 306 cGy (90\%), blue line 272 cGy (80\%). $4 b^{*}$ : Patient 4 refused follow-up photography. Images do not necessarily correlate with the last follow-up date but represent the latest available images from each patient 
Table 2. Dosimetric characteristics of the seven patients treated with brachytherapy-based accelerated partial breast irradiation (B-APBI) with existing breast implants

\begin{tabular}{lccccccccccc} 
& $\begin{array}{c}\text { PTV_EVAL } \\
\text { volume (cc) }\end{array}$ & $\begin{array}{c}\text { Device to } \\
\text { implant } \\
\text { distance } \\
(\mathrm{mm})\end{array}$ & $\begin{array}{c}\text { Implant } \\
\text { volume } \\
(\mathrm{cc})\end{array}$ & $\begin{array}{c}\text { Mean } \\
\text { dose to } \\
\text { implant } \\
(\mathrm{Gy})\end{array}$ & $\begin{array}{c}\text { Maximum } \\
\text { dose to } \\
\text { implant } \\
(\mathrm{Gy})\end{array}$ & $\begin{array}{c}\text { Implant } \\
\mathrm{V}_{50}(\%)\end{array}$ & $\begin{array}{c}\text { Implant } \\
\mathrm{V}_{75}(\%)\end{array}$ & $\begin{array}{c}\text { Implant } \\
\mathrm{V}_{100}(\%)\end{array}$ & $\begin{array}{c}\text { Implant } \\
\mathrm{V}_{150}(\%)\end{array}$ & $\begin{array}{c}\text { Implant } \\
\mathrm{V}_{200}(\%)\end{array}$ \\
\hline Patient \#1 & 69.9 & 1.3 & 238.3 & 17.37 & 90.2 & 18.1 & 8.4 & 4.2 & 1.1 & 0.15 \\
\hline Patient \#2 & 73.9 & 1 & 234.5 & 15.4 & 72.8 & 31.1 & 16.5 & 8.6 & 2.0 & 0 \\
\hline Patient \#3 & 38.7 & 1 & 379.2 & 11.7 & 232.3 & 8.9 & 4.4 & 2.4 & 0.8 & 0.1 \\
\hline Patient \#4 & 41.9 & 1.5 & 389.8 & 9.5 & 211.7 & 5.9 & 2.7 & 1.5 & 0.5 & 0.2 \\
\hline Patient \#5 & 29.1 & 1 & 355.1 & 6.1 & 126.3 & 6.0 & 2.5 & 0.3 & 0.2 & 0.1 \\
\hline Patient \#6 & 44.6 & 1.3 & 241.7 & 16.7 & 442.3 & 15.6 & 9.3 & 5.8 & 2.3 & 0.3 \\
\hline Patient \#7 & 93.7 & 1 & 468.3 & 23.1 & 535.1 & 23.9 & 15 & 9.5 & 4.1 & 1.9
\end{tabular}

$V_{50}, V_{75}, V_{100}, V_{150}, V_{200}$ represent the percentage of breast implant volumes receiving $50 \%, 75 \%, 100 \%, 150 \%$, and $200 \%$ of the prescribed dose. All maximum doses were point doses to less than $1 \mathrm{cc}$ of breast implant volume

Table 3. Correlation of absolute and relative dose to existing breast implants and correlation with cosmetic outcome

\begin{tabular}{lcccccccccccc} 
& $\begin{array}{c}\text { Implant } \\
\text { volume } \\
(\mathrm{cc})\end{array}$ & $\mathrm{V}_{50}(\%)$ & $\mathrm{V}_{50}{ }^{\prime}(\mathrm{cc})$ & $\mathrm{V}_{75}(\%)$ & $\mathrm{V}_{75}{ }^{\prime}(\mathrm{cc})$ & $\mathrm{V}_{100}(\%)$ & $\mathrm{V}_{100}{ }^{\prime}(\mathrm{cc})$ & $\mathrm{V}_{150}(\%)$ & $\mathrm{V}_{150}{ }^{\prime}(\mathrm{cc})$ & $\mathrm{V}_{200}(\%)$ & $\mathrm{V}_{200}$ (cc) & Cosmesis \\
\hline Patient \#1 & 238.3 & 18.1 & 43.1 & 8.4 & 20 & 4.2 & 10 & 1.1 & 2.6 & 0.15 & 0.3 & Excellent \\
\hline Patient \#2 & 234.5 & 31.1 & 72.9 & 16.5 & 38.7 & 8.6 & 20.2 & 2.0 & 4.7 & 0 & 0 & Good \\
\hline Patient \#3 & 379.2 & 8.9 & 33.7 & 4.4 & 16.7 & 2.4 & 9.1 & 0.8 & 3 & 0.1 & 0.4 & Excellent \\
\hline Patient \#4 & 389.8 & 5.9 & 22.9 & 2.7 & 10.5 & 1.5 & 5.9 & 0.5 & 1.9 & 0.2 & 0.8 & Excellent \\
\hline Patient \#5 & 355.1 & 6.0 & 21 & 2.5 & 8.9 & 0.3 & 1.1 & 0.2 & 0.7 & 0.1 & 0.4 & Excellent \\
\hline Patient \#6 & 241.7 & 15.6 & 37.7 & 9.3 & 22.5 & 5.8 & 14 & 2.3 & 5.6 & 0.8 & 1.9 & Excellent \\
\hline Patient \#7 & 468.3 & 23.9 & 111.9 & 15 & 70.2 & 9.5 & 44.5 & 4.1 & 19.2 & 1.9 & 8.9 & Fair
\end{tabular}

$V_{50}, V_{75}, V_{100}, V_{150}, V_{200}$ represent the percentage of breast implant volumes receiving $50 \%, 75 \%, 100 \%, 150 \%$, and $200 \%$ of the prescribed dose. $V_{50}, V_{75}^{\prime}, V_{100}, V_{150}$, and $V_{200}$ 'represent the absolute volume of the breast implant receiving $50 \%, 75 \%, 100 \%, 150 \%$, and $200 \%$ of the prescribed dose, respectively

margin would have been nearly impossible to obtain, since there were no remaining tissue except the implant itself. B-APBI in those cases allowed those patients to maintain their existing breast implants with excellent cosmetic outcomes. The majority of the other patients also had excellent or good cosmetic outcome, except patient \#7, who had a sizeable difference between her breasts even prior to start of her B-APBI and also has now experienced rupture of her implant on the treated side. Importantly, all other women were able to maintain their breast implants except patient \#5 who had to undergo replacement of her implants bilaterally due to age-related leakage. There were no grade 3 or 4 late toxicities and all patients tolerated the treatments well.

In terms of dose received by the breast implants, our values of mean dose of $12.1 \mathrm{~Gy}, \mathrm{~V}_{50}$ of $15.6 \%, \mathrm{~V}_{75}$ of $7 \%$, $\mathrm{V}_{100}$ of $4.6 \%, \mathrm{~V}_{150}$ of $1.58 \%$, and $\mathrm{V}_{200}$ of $0.46 \%$ may represent benchmarks to be used for future treatment planning in patients with breast implants. Overall, the lower the dose to the implant and the smaller the absolute implant volume treated, the more beneficial this appears to be in

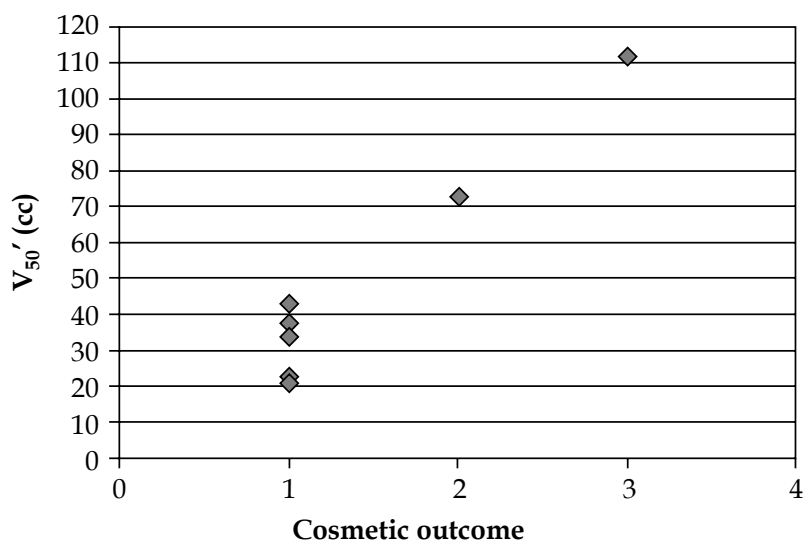

Fig. 2. $\mathrm{V}_{50}{ }^{\prime}(\mathrm{cc})$ corresponding to each patient's cosmetic outcome. Outcomes based on the Harvard scale, 1 - excellent, 2 - good, 3 - fair, and 4 - poor. $\mathrm{V}_{50}{ }^{\prime}$ (cc) represents the absolute volume of breast implant that received $50 \%$ of the prescribed dose. All patients with excellent cosmetic outcome had $\mathrm{V}_{50}{ }^{\prime}(\mathrm{cc})$ of less than 50 
Table 4. Comparison of studies reporting treatment with accelerated partial breast irradiation (APBI) in the setting of breast implants

\begin{tabular}{lccccc} 
Study & $\begin{array}{c}\text { Total \# of treated } \\
\text { patients }\end{array}$ & $\begin{array}{c}\text { \# of patients treated } \\
\text { with multi-lumen or } \\
\text { catheter devices }\end{array}$ & $\begin{array}{c}\text { Median } \\
\text { follow-up }\end{array}$ & $\begin{array}{c}\text { Excellent/good } \\
\text { cosmetic } \\
\text { outcome }\end{array}$ & $\begin{array}{c}\text { Median } \mathrm{V}_{50} \\
\text { to the breast } \\
\text { implant }\end{array}$ \\
\hline Kuske et al. [12] & 87 & 6 & 36 months & Not reported \\
\hline Bloom et al. [13] & 1 & 1 & $67 \%$ months & $100 \%$ & 83.3 cc \\
\hline Current study & 7 & 7 & 32 months & 33.04 cc
\end{tabular}

terms of late cosmetic outcome. As a result, B-APBI might be a better option compared to external beam radiation as well in patients who wish to maintain their implants since a smaller overall volume is treated. The correlation between volume of implant treated and cosmesis was apparent in our two patients who had the highest $V_{50}$ ' along with the worst cosmetic outcomes. Additionally, patient \#7 who had the highest mean, maximum, and absolute and relative dose to the implant went on to experience intra and extracapsular rupture of her implant, raising the possibility that there might exist an absolute implant dose tolerance. All of the patients with $\mathrm{V}_{50}$ ' of less than $50 \mathrm{cc}$ had an excellent cosmetic outcome (Figure 2), although this could potentially be a marker for overall lower dose to the implant. Our patient with the worst cosmetic outcome also had the highest maximum and mean dose to the breast implant. Furthermore, the distance from the device to the implant did not appear to have a significant impact on the cosmetic outcome as most devices were within 1-2 mm of the breast implants. We acknowledge that a larger population with longer follow-up is needed to confirm these findings.

\section{Conclusions}

Although the small number of patients in our series along with our limited follow-up limits our findings, we have nevertheless shown that B-APBI in patients with adjacent pre-existing breast implants is both safe and efficacious with good to excellent cosmetic outcomes in early clinical follow-up. Ideally, the dose to the breast implants should be lowered as much as possible during the treatment planning process. Based on the above results, unnecessary dose raises the possibility of future poorer cosmetic outcome and other complications like implant rupture. Therefore, we believe that there is no need to expand the PTV_EVAL uniformly into the breast implant, since it contains no actual breast tissue and that PTV_EVAL can be pulled back as long as all of the necessary breast tissue is treated. Additionally, the $\mathrm{V}_{50}$ ' can be used as a marker of appropriate amount of dose to the implant, with $50 \mathrm{cc}$ or less receiving $50 \%$ of prescribed dose representing a reasonable marker. Our other reported values of mean dose and the $\mathrm{V}_{50}, \mathrm{~V}_{75}, \mathrm{~V}_{100}, \mathrm{~V}_{150}$, and $\mathrm{V}_{200}$ may be used as benchmarks to decrease the dose and any risks of possible complications. These parameters will need to be confirmed in a larger series with long-term follow-up. In the meantime, our dosimetric parameters have the added benefit of theoretically reducing the chances of capsular contracture, which is the most feared cosmetic complication in women with augmented breasts who undergo external beam whole breast irradiation [19]. Additionally, we have shown much better cosmetic outcomes compared to previously reported cases of whole breast irradiation in patients with existing breast implants, in which all twenty one patients were reported to have fair or poor cosmetic results [20]. Therefore, we conclude that with careful consideration during treatment planning and attention to tolerance of breast implants, B-APBI can be an excellent option for early stage breast cancer patients with augmented breasts who need to undergo adjuvant radiation therapy.

\section{Disclosure}

Authors report no conflict of interest.

\section{References}

1. Veronesi U, Cascinelli N, Mariani L et al. Twenty-year follow-up of a randomized study comparing breast-conserving surgery with radical mastectomy for early breast cancer. N Engl J Med 2002; 347: 1227-1232.

2. NCCN clinical practice guidelines [online]. Retrieved from http://www.nccn.org/professionals/physician_gls/pdf/ breast.pdf (access: 30.06.2015).

3. Skowronek J, Chichel A. Brachytherapy in breast cancer: an effective alternative. Prz Menopauzalny 2014; 13: 48-55.

4. Vicini FA, Antonucci JV, Wallace M et al. Long-term efficacy and patterns of failure after accelerated partial breast irradiation: a molecular assay-based clonality evaluation. Int J Radiat Oncol Biol Phys 2007; 68: 341-346.

5. Koh VY, Buhari SA, Tan PW et al. Comparing a volume based template approach and ultrasound guided freehand approach in multicatheter interstitial accelerated partial breast irradiation. J Contemp Brachytherapy 2014; 6: 173-177.

6. Smith GL, Huo J, Giordano SH et al. Utilization and outcomes of breast brachytherapy in younger women. Int J Radiat Oncol 2015; 93: 91-101.

7. Shah C, Vicini F, Wazer DE et al. The American Brachytherapy Society consensus statement for accelerated partial breast irradiation. Brachytherapy 2013; 12: 267-277.

8. The American Society of Breast Surgeons. Consensus Statement for Accelerated Partial Breast Irradiation [online]. Retrieved from: https://www.breastsurgeons.org/statements/PDF_Statements/APBI.pdf (access: 30.06.2015).

9. Smith BD, Arthur DW, Buchholz TA et al. Accelerated partial breast irradiation consensus statement from the American Society for Radiation Oncology (ASTRO). Int J Radiat Oncol Biol Phys 2009; 74: 987-1001.

10. Polgár C, Van Limbergen E, Pötter R et al. Patient selection for accelerated partial-breast irradiation (APBI) after 
breast-conserving surgery: recommendations of the Groupe Europeen de Curietherapie-European Society for Therapeutic Radiology and Oncology (GEC-ESTRO) breast cancer working group based on clinical evidence (2009). Radiother Oncol 2010; 94: 264-273.

11. American Society of Plastic Surgeons. 2012 Plastic Surgery Statistics Report [online]. Retrieved from: http://www. plasticsurgery.org/Documents/news-resources/statistics/2012-Plastic-Surgery-Statistics/full-plastic-surgery-statistics-report.pdf (access: 01.08.2015).

12. Kuske R, Chowdhury R, Snyder M et al. Accelerated partial breast irradiation for select breast cancers in the presence of augmentation. Int J Radiat Oncol Biol Phys 2012; 84: S87 [Abstract].

13. Bloom ES, Kirsner S, Mason BE et al. Accelerated partial breast irradiation using the strut-adjusted volume implant single-entry hybrid catheter in brachytherapy for breast cancer in the setting of breast augmentation. Brachytherapy 2011; 10: $178-183$.

14. Akhtari M, Nitsch PL, Bass BL, Teh BS. Long-term outcome of accelerated partial breast irradiation using a multilumen balloon applicator in a patient with existing breast implants. Brachytherapy 2015; 14: 289-292.

15. Kuo H, Mehta KJ, Hong L et al. Day to day treatment variations of accelerated partial breast brachytherapy using a multi-lumen balloon. J Contemp Brachytherapy 2014; 6: 68-75

16. Harris JR, Levene MB, Svensson G et al. Analysis of cosmetic results following primary radiation therapy for stages I and II carcinoma of the breast. Int J Radiat Oncol Biol Phys 1979; 5: 257-261.

17. Polgár C, Fodor J, Major T et al. Breast-conserving therapy with partial or whole breast irradiation: ten-year results of the Budapest randomized trial. Radiother Oncol 2013; 108: 197-202.

18. Kuske R. Breast conservation therapy without capsular contracture in young augmented women using interstitial brachytherapy. J Contemp Brachytherapy 2014; 6: 231-235.

19. Fodor J, Gulyas G, Polgár $C$ et al. Radiotherapy and breast reconstruction: the issue of compatibility. Orv Hetil 2003; 144: $549-555$.

20. Mark RJ, Zimmerman RP, Greif JM. Capsular contracture after lumpectomy and radiation therapy in patients who have undergone uncomplicated bilateral augmentation mammoplasty. Radiology 1996; 200: 621-625. 